\title{
IDENTIFICACIÓN Y CUANTIFICACIÓN DE PÉRDIDAS DE SACAROSA EN EL EFLUENTE FINAL DEL PROCESO DE ELABORACIÓN DE AZÚCAR EN EL INGENIO AZUCARERO RiOPAILA CASTILlA (Planta Castilla)
}

\author{
Efrén Corrales-Correa ${ }^{1}$, Guillermo Garzón-García ${ }^{2}$
}

${ }^{1}$ Jefe de Producción en el Ingenio Riopaila Castilla. Correo electrónico: ecorrales@riopaila-castilla.com 2 Profesor de la Facultad de Ciencias Básicas, Programa de Química, Universidad Santiago de Cali, Cali, Colombia

Recibido: 28 de agosto del 2014. Aprobado: 16 de octubre del 2014.

Cómo citar este artículo: E. Corrales-Correa y G. Garzón-García, "Identificación y cuantificación de pérdidas de sacarosa en el efluente final del proceso de elaboración de azúcar en el ingenio azucarero Ríopaila Castilla (planta Castilla)”. Ingeniería Solidaria, vol. 10, n. ${ }^{\circ}$ 17, pp. 83-91, en.-dic., 2014. doi: http://dx.doi. org/10.16925/in.v9i17.808

Resumen. El artículo es producto del proyecto de grado titulado "Identificación y cuantificación de pérdidas de sacarosa en el efluente final del proceso de elaboración de azúcar en un ingenio azucarero" desarrollado en la Universidad Santiago de Cali. El objetivo del proyecto fue la identificación y cuantificación de pérdidas de sacarosa en el efluente de elaboración del Ingenio Riopaila Castilla, planta Castilla. El problema de investigación surgió del interés en aprovechar la gran cantidad de sacarosa que se pierde en el proceso de elaboración del azúcar con un valor aproximado de \$1500 millones por año. Los resultados de investigación fueron: una disminución de pérdidas de sacarosa que posiciona al ingenio en el primer lugar con las pérdidas de sacarosa más bajas de todo el sector azucarero en Colombia; la creación de seis nuevos puestos de trabajo (inspectores de pérdidas); disminución del impacto ambiental con la reducción de sacarosa contaminante en el efluente final; y un mejor manejo en el control de la sacarosa en el efluente final.

Palabras clave: sacarosa, efluente, brix, jugo clarificado.

IDENTIFICATION AND QUANTIFICATION OF SUCROSE LOSSES in the Final Effluent of the Sugar-making Process at the Riopaila Castilla Sugar Mill (Castilla plant)

Abstract. This article stems from the graduate project "Identification and Quantification of Sucrose Losses in the Final Effluent of the Sugar-making Process at a Sugar Mill" ("Identificación y cuantificación de pérdidas de sacarosa en el efluente final del proceso de elaboración de azúcar en un ingenio azucarero") carried out at the Universidad Santiago de Cali. The objective was to identify and quantify sucrose losses in the manufacturing effluent at the Castilla plant of the Riopaila Castilla sugar mill. The research problem stemmed from interest in making use of the large quantity of sucrose lost during the sugar making process, amounting to approximately 1.5 billion COP per year. The results were a decrease in sucrose losses that earned the mill first place, with the lowest sucrose losses in the entire Colombian sugar sector; the creation of six new jobs (losses inspectors); a decrease in environmental impact through reduction of polluting sucrose in the final effluent; and better handling to control sucrose in the final effluent.

Keywords: sucrose, flow, brix, clarified juice.

\section{IDENTIFICAÇÃo E QUANTIFICAÇÃo DE PERDAS DE SACAROSE NO EFLUENTE FINAL DO PROCESSO DE ELABORAÇÃO DE AÇÚCAR NA USINA AÇUCAREIRA Riopaila Castilla (Fábrica Castilla)}

Resumo. Este artigo é produto do trabalho de conclusão de curso intitulado "Identificação e quantificação de perdas de sacarose no efluente final do processo de elaboração de açúcar numa usina açucareira", desenvolvido na Universidade de Santiago de Cali. O objetivo do projeto foi a identificação e quantificação de perdas de sacarose no efluente de elaboração da Usina Riopaila Castilla, fábrica Castilla. O problema da pesquisa surgiu do interesse em aproveitar a grande quantidade de sacarose que se perde no processo de elaboração do açúcar com um valor aproximado a \$1500 milhões por ano. Os resultados de pesquisa foram: uma diminuição de perdas de sacarose que posiciona a usina no primeiro lugar com as perdas de sacarose mais baixas de todo o setor açucareiro na Colômbia; a criação de seis novos postos de trabalho (inspetores de perdas); diminuição do impacto ambiental com a redução de sacarose contaminante no efluente final; e uma melhor gestão no controle da sacarose no efluente final.

Palavras-chave: sacarose, efluente, brix, suco clarificado. 


\section{Introducción}

La industria azucarera es uno de los sectores económicos de mayor importancia en nuestro país. Su producción comienza en el campo con la adecuación y preparación de las tierras, la siembra y el sostenimiento de la plantación de la caña de azúcar. Cuando la caña está lista para el corte se da inicio a la operación cosecha y se lleva a la fábrica para el proceso de extracción y cristalización de la sacarosa. Este proceso se divide en ocho etapas: molienda, clarificación, filtración, evaporación, cocimiento, centrifugación, secado y empacado.

En el proceso de fabricación del azúcar crudo se presentan usualmente pérdidas de materia primas que representan una considerable pérdida financiera para un ingenio azucarero. No es de extrañar que un aspecto fundamental para analizar el rendimiento de una fábrica de azúcar sea contabilizar el nivel de pérdidas de las materias primas. Entre las mayores pérdidas está la pérdida de la sacarosa. Guzmán Jaimes [1], en su trabajo de grado titulado "Identificación de las principales pérdidas de sacarosa y estrategias a implementar para aumentar la eficiencia en el proceso de elaboración de azúcar", brinda una definición sencilla de la sacarosa: "es un carbohidrato disacárido producido por la condensación de la glucosa y la fructuosa y tiene la fórmula empírica: $\mathrm{C}_{12} \mathrm{H}_{22} \mathrm{O}_{11}$ ".

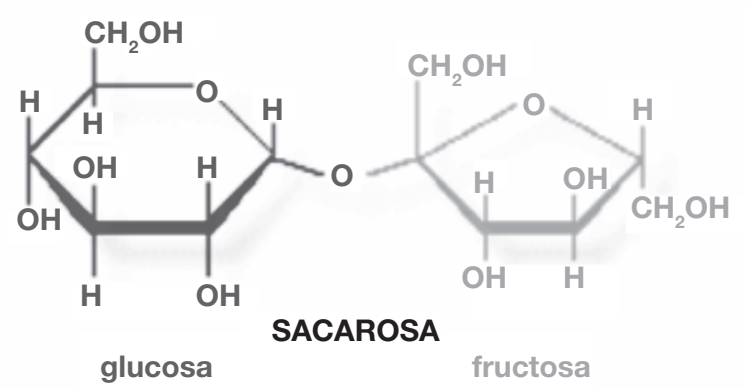

Figura 1. Fórmula química de la sacarosa Fuente: [1]

El investigador colombiano Jesús Laharrondo ha sido uno de los pioneros en el estudio de la pérdida de la sacarosa. Larrahondo expresa que "el deterioro de la caña y la pérdida de sacarosa entre el corte y la molienda han sido objeto de varios estudios, bajo las condiciones del Valle del Cauca (Colombia) y en diferentes países o sectores azucareros. La tasa de deterioro depende de las condiciones ambientales, de la variedad y del sistema de cosecha. Cuando el corte es mecánico, el deterioro es mayor, debido al incremento de las infecciones de origen bacteriano en los tallos" [2].
Laharrondo [2] refiere que "estudios realizados por Cenicaña (Centro de Investigación de la Caña de Azúcar de Colombia) [3] demostraron que por cada unidad (en porcentaje) de materia extraña puede reducir el rendimiento o niveles de sacarosa entre 0,14 y 0,20 unidades porcentuales. Además, de las pérdidas o reducciones en sacarosa, la presencia de $1 \%$ de materia extraña puede ocasionar incrementos entre $0,1(\%)$ y 0,8 (\%) en la fibra \% caña”.

La mayoría de estudios reportados por Cenicaña se centran en pérdidas de sacarosa en etapas de corte, alce y transporte de la caña que lo afectan los siguientes condiciones: tiempo de permanencia, pérdidas por quema, pérdidas por materia extraña, mediciones realizadas directamente en la caña (método polarimétrico y refracto métrico). Las pérdidas de nuestra investigación son de sacarosa que ya ha sido extraída y se tiene ya en el proceso fabril, y para ella se aplican métodos analíticos diferentes (análisis colorimétricos).

Se han publicado numerosos estudiosos sobre las pérdidas de sacarosa, entre los cuales resaltan los siguientes: "Evaluación, análisis y cuantificación de pérdidas de sacarosa de miel final en los procesos de cristalización, agotamiento y centrifugación del Ingenio María Luisa" [4], "Propuesta de alternativas para la reducción de pérdidas de sacarosa de un ingenio azucarero [5] y "Participación en la acción microbiológica en las pérdidas indeterminadas de sacarosa" [6]. Se resalta el gran número de investigaciones reportadas en la base de datos de Cenicaña, las cuales son un punto de partida para las nuevas investigaciones.

\section{Pérdidas del sector azucarero}

Generalmente, las pérdidas en el sector azucarero se clasifican en dos tipos: determinadas e indeterminadas. "Las pérdidas determinadas son aquellas que se pueden controlar, cuantificar y calificar, son originadas por el bagazo, la cachaza y la miel final" [4]. Dentro de las pérdidas indeterminadas, tienen lugar las pérdidas químicas, ya sea por inversión o descomposición de la sacarosa, o más generalmente, una combinación de las dos, en que el control de rutina puede determinar el fenómeno pero no puede determinar las pérdidas por medios químicos. De igual forma, el azúcar que pasa a las mieles se considera como pérdida aun cuando constituye parte de un subproducto vendible y se registra totalmente en el control. Estas pérdidas indeterminadas, representan pérdidas reales que ocurren durante el proceso, las 
cuales no se pueden relacionar directamente con un área específica y pueden reflejar errores en pesos, análisis o inversiones.

Por otra parte, existen unas llamadas pérdidas aparentes, las cuales son no reales pero representan un aspecto importante en el informe de las pérdidas de una fábrica y son causadas por algún error en los pesos de caña, el jugo, el azúcar o se deben a errores de análisis o estimaciones incorrectas en la determinación de las existencias. Estas pérdidas son reducibles a un mínimo mediante la cuidadosa supervisión de los pesos, pruebas y métodos. Sin embargo, estas pérdidas aparentes al operar en forma contraria, encubren pérdidas verdaderas y hacen que las cifras de la fábrica aparezcan mejor de lo que realmente son. Un ejemplo de ello sería que los errores de muestreo, pesaje o análisis del jugo mixto tiendan a mostrar menos jugo o menos azúcar en este.

Las anteriores pérdidas dan lugar a las pérdidas totales, que son la suma de cada una de las pérdidas generadas en las estaciones fabriles e involucran pérdidas por sacarosa en bagazo, cachaza, miel final, provenientes de pérdidas mecánicas (determinadas), y aquellas provenientes de pérdidas por descomposición, las cuales se generan por acción de ácidos o sales ácidas que convierten la sacarosa en una mezcla de glucosa, dextrosa y levulosa llamada azúcar invertido (indeterminadas). Sin embargo, en esta sumatoria de pérdidas totales, se encuentra un tipo de pérdidas que corresponde a las indeterminadas y tienen lugar a la pérdida de sacarosa en aguas de efluentes y está asociada al área de elaboración.

De lo anterior, se observa una problemática en materia de control de pérdidas de sacarosa en los ingenios que conllevan consecuencias financieras importantes. Adicionalmente, otro motivo por el cual las administraciones de los ingenios no prestan mucha atención a este tipo de pérdidas, se debe a que el control inicia después de que el guarapo se ha extraído; sin embargo, las grandes pérdidas ocurren desde el momento en que se corta la caña hasta el momento en que se muele. Es por esto que los funcionarios supervisores deben mantener siempre en mente estas dos posibilidades de pérdida y reconocer que una atención más estrecha a ellas puede conducir a ahorros financieros considerables en los materiales, equipos y en la parte operacional, donde los efectos son más drásticos.

La finalidad de este trabajo es identificar, cuantificar y conocer el efecto económico de las pérdidas, y a partir de esto formular soluciones en el proceso de producción que permitan optimizar el control tanto químico como operacional y mecánico del ingenio. Asímismo, se intenta minimizar las pérdidas indeterminadas y, por consiguiente, las pérdidas totales, las cuales se están vertiendo por las cañerías normalmente. Esta investigación contó con los equipos aforadores de caudal y sistemas de recuperación. Se pretendió cuantificar estas pérdidas indeterminadas de sacarosa con mayor precisión y con la ayuda también de un método analítico confiable, lo cual supuso una revisión muy detallada a cada una de las aguas que están siendo vertidas al efluente final y reubicar las aguas que no aportan sacarosa al efluente.

\section{Metodología}

Se utilizó una metodología Sugar Cane [7], [8] que permitió determinar las pérdidas de sacarosa presente en aguas de efluente de elaboración, vertida a este por distintas causas, además de establecer un sistema documentado de identificación y cuantificación de pérdidas.

\subsection{Principio del método}

El método consiste en formar un complejo entre fenol y sacarosa en medio ácido. El color desarrollado es medido en un espectrofotómetro a $490 \mathrm{~nm}$ y comparado con una curva de calibración construida previamente. El muestreo es realizado por medio de una bomba neumática de flujo continuo, con una frecuencia de recolección de cada hora. Estas muestras son llevadas al laboratorio y se forma el complejo con las muestras recolectadas durante dos horas. El punto de muestreo es el canal de desagüe de las aguas de elaboración ubicado en frente de las calderas donde se instaló un medidor de caudal para poder tomar el aforo al momento de la toma de la muestra.

\subsection{Procedimiento}

Previo al análisis de las muestras, se construyó una curva de calibración con los espectrofotómetros empleados [7]. Esta calibración se realizó en dos equipos diferentes cuyos resultados finales fueron muy similares; el objetivo de realizar estas dos curvas en dos equipos diferentes era el de tener un plan alternativo que permitiera garantizar la continuidad de los análisis en caso de falla. Para realizar esta curva de calibración, se empleó como solución estándar una solución de azúcar refinado en concentraciones de 10 a 100 ppm y una longitud de onda de $490 \mathrm{~nm}$.

Los resultados obtenidos por uno de los equipos se muestran en la tabla 1. 
Tabla 1. Datos curva de calibración espectrofotómetro Génesys 10 visible

\begin{tabular}{|c|c|}
\hline $\begin{array}{c}\text { Concentración } \\
(\mathrm{ppm})\end{array}$ & Absorbancia \\
\hline 10 & 0,091 \\
\hline 20 & 0,237 \\
\hline 30 & 0,355 \\
\hline 40 & 0,489 \\
\hline 50 & 0,578 \\
\hline 100 & 1,108 \\
\hline
\end{tabular}

Pendiente: 0,0111; Corte en $x$ : 0,0134; Coeficiente de correlación: 0,9961 Fuente: elaboración propia

\subsection{Proceso de análisis}

El procedimiento para el análisis fue como sigue: filtrar la muestra si está muy turbia; pipetear $2 \mathrm{~mL}$ de muestra a un balón aforrado de $100 \mathrm{~mL}$; tomar $2 \mathrm{~mL}$ de la muestra diluida en un tubo de ensayo; agregar $1 \mathrm{~mL}$ de solución de fenol al 5\% y agitar fuertemente; añadir desde la bureta $5 \mathrm{~mL}$ de ácido sulfúrico concentrado de tal forma que reaccione con la muestra; agitar suavemente y dejar la solución a temperatura ambiente durante 20 minutos; enfriar con agua y agitar fuertemente; leer la absorbancia en el espectrofotómetro a $490 \mathrm{~nm}$ en una celda de $10 \mathrm{~mm}$, utilizando como cero agua destilada; simultáneamente preparar un blanco por sustitución de los $2 \mathrm{~mL}$ de muestra por agua; hacer la lectura de absorbancia del blanco.

\subsection{Instrumentos, materiales y reactivos}

Se utilizaron los siguientes: muestreador automático (SANDPIPER); recipiente plástico, capacidad 2L; aforador (Canaleta Parshall); sistema de refrigeración de muestra; espectrofotómetros UV/Vis Gennesys y Espectronic 20D; balanza analítica 0,1g Mettler Toledo, AB204-s; solución de fenol al 5\%; acido sulfúrico concentrado; solución estándar de azúcar refinado; pH metro Schott.

\section{Resultados y análisis}

Los resultados obtenidos demuestran que gran cantidad de sacarosa se perdía por el efluente final de elaboración en tiempos anteriores al segundo semestre del año 2007, fecha en la cual entró en funcionamiento el Sistema Integrado de Recuperación de Sacarosa.

La figura 2 resume el comportamiento histórico de las pérdidas de sacarosa \% caña en indeterminadas y totales a partir del año 2006, mostrando la tendencia y proporcionalidad de las pérdidas por efluente con las pérdidas indeterminadas y estas a su vez con las pérdidas totales.

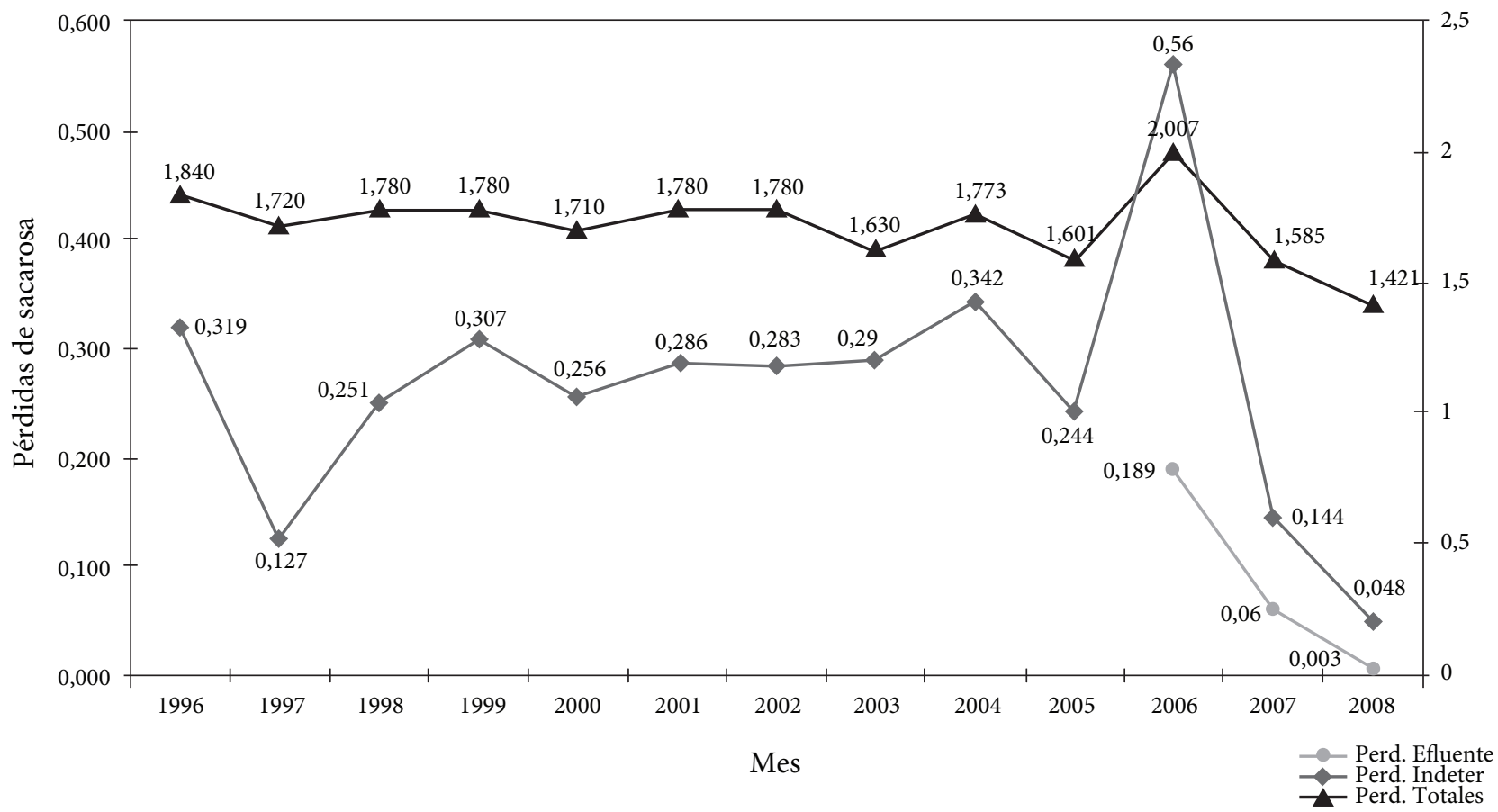

Figura 2. Histórico de pérdidas de sacarosa \% caña efluente, indeterminadas y totales. Año 1996-2008 
En la figura 2, se muestra la tendencia del comportamiento de las pérdidas de sacarosa desde el año 1996 al 2008, y la notable disminución de las pérdidas indeterminadas y totales por efecto de la reducción de las pérdidas por efluente final de elaboración.
La tabla 2 muestra la disminución de los niveles de pérdidas totales, indeterminadas \% caña del efluente de elaboración a partir del segundo semestre del año 2007.

Tabla 2. Pérdidas de sacarosa efluente frente a las pérdidas totales e indeterminadas, 2007

\begin{tabular}{|c|c|c|c|c|}
\hline Año & Mes & $\begin{array}{l}\text { Pérdidas indeterminadas } \\
\text { Sac \% caña }\end{array}$ & $\begin{array}{l}\text { Pérdidas totales Sac \% } \\
\text { caña }\end{array}$ & $\begin{array}{l}\text { Pérdidas efluente Sac \% } \\
\text { caña }\end{array}$ \\
\hline \multirow{12}{*}{2007} & Enero & 0,510 & 1,939 & 0,126 \\
\hline & Febrero & 0,273 & 1,751 & 0,167 \\
\hline & Marzo & 0,236 & 1,730 & 0,064 \\
\hline & Abril & 0,064 & 1,508 & 0,053 \\
\hline & Mayo & 0,018 & 1,482 & 0,069 \\
\hline & Junio & 0,145 & 1,481 & 0,020 \\
\hline & Julio & 0,061 & 1,512 & 0,022 \\
\hline & Agosto & 0,071 & 1,628 & 0,038 \\
\hline & Septiembre & 0,006 & 1,407 & 0,012 \\
\hline & Octubre & 0,110 & 1,454 & 0,014 \\
\hline & Noviembre & 0,009 & 1,395 & 0,050 \\
\hline & Diciembre & 0,012 & 1,401 & 0,013 \\
\hline Total & Año & 0,126 & 1,557 & 0,054 \\
\hline
\end{tabular}

Fuente: datos históricos de producción Ingenio

Por otra parte, las pérdidas económicas por derrames de sacarosa al efluente en el segundo semestre del 2006 ascendieron a 1777 millones de pesos y en el 2007 fueron de 1088 millones de pesos, con una notable disminución en el segundo semestre del mismo año. Los valores en el 2008 fueron muy bajos, alcanzando un valor de 49,4 millones de pesos, en el ingenio Riopaila-Castilla, planta Castilla, tal como se muestra en la tabla 3.

Tabla 3. Pérdidas de sacarosa efluente frente a quintales y pesos, 2008

\begin{tabular}{|c|c|c|c|c|}
\hline Año & Mes & Azúcar (qq) & $\begin{array}{l}\text { Pérdidas efluente Sac \% } \\
\text { Caña }\end{array}$ & Pérdidas pesos (Millones) \\
\hline \multirow{11}{*}{2008} & Enero & 109 & 0,004 & $\$ 5232000$ \\
\hline & Febrero & 161 & 0,006 & $\$ 7728000$ \\
\hline & Marzo & 225 & 0,006 & $\$ 10800000$ \\
\hline & Abril & 33 & 0,002 & $\$ 1584000$ \\
\hline & Mayo & 52 & 0,002 & $\$ 2496000$ \\
\hline & Junio & 80 & 0,002 & $\$ 3840000$ \\
\hline & Julio & 47 & 0,001 & $\$ 2256000$ \\
\hline & Agosto & 46 & 0,001 & $\$ 2208000$ \\
\hline & Septiembre & 112 & 0,006 & $\$ 5376000$ \\
\hline & Noviembre & 34 & 0,001 & $\$ 1632000$ \\
\hline & Diciembre & 131 & 0,003 & $\$ 6288000$ \\
\hline Total & Año & 1030 & 0,003 & $\$ 49440000$ \\
\hline
\end{tabular}

Fuente: elaboración propia 


\subsection{Mejoramiento en el sistema de recuperación de sacarosa en efluentes}

Uno de los logros obtenidos en el presente trabajo fue el mejoramiento del sistema de recuperación de aguas dulces, implementando acciones correctivas en el canal de recuperación, como lo son: el tanque de recuperación de crudo instalado en acero inoxidable con su respectivo sistema de bombeo independiente; instalación de tanque de recuperación de la refinería en acero inoxidable; reposición de tanque de disolución de azúcar lado empacadero de acero al carbón a acero inoxidable, los cuales combinaban las aguas dulces con flujos de aguas sin dulce (aguas de enfriamiento de equipos), ocasionando excesivo volumen y diluyendo la sacarosa dificultando su recuperación, altos tiempos de residencia y degradación de la sacarosa.

La figura 3 muestra la canaleta Parshall junto con la bomba neumática de recolección y el punto de muestreo implementados para cuantificar las pérdidas de sacarosa.

La canaleta Parshall es empleada como canal de rechazo de las aguas que no deben contener sacarosa, como medida de separación con las aguas dulces las que se recuperan por un canal alterno o directamente en el tanque.

En el proceso fabril de la caña de azúcar, las pérdidas físicas son el principal causante de las pérdidas indeterminadas y la inversión de la sacarosa ocupa el

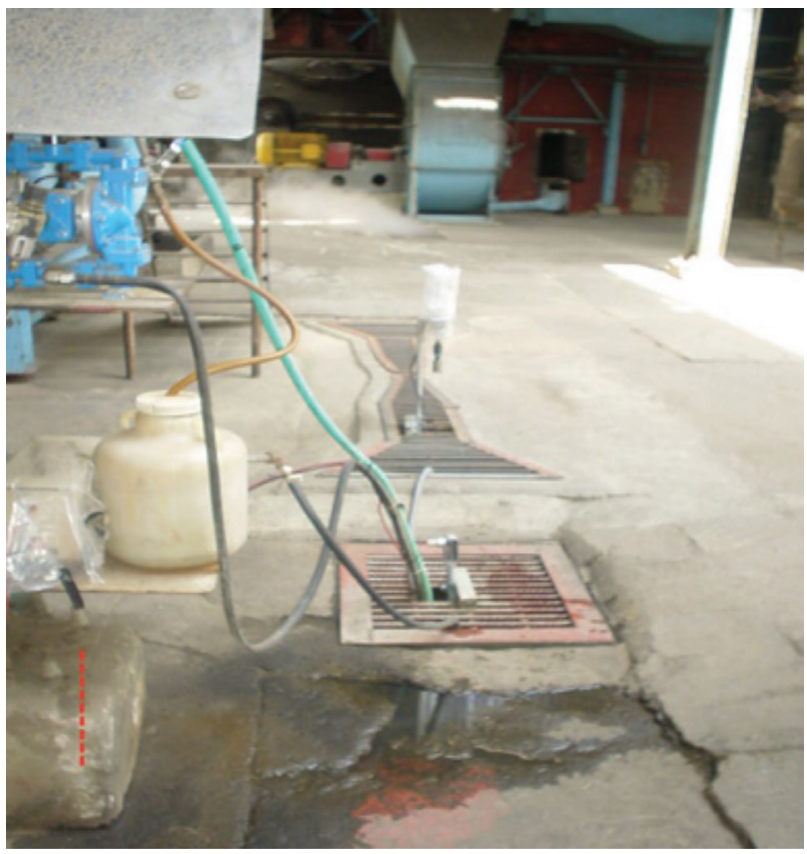

Figura 3. Toma de muestra, tarro recolector, bomba neumática y al fondo canaleta Parshall Fuente: elaboración propia segundo lugar, por lo cual fue necesario desarrollar una optimización del sistema que consistió en:

- corrección de fugas de material azucarado en la refinería,

- identificación de los puntos por donde se perdía y plan de recuperación,

- clasificación de drenajes en aguas dulces y no dulces,

- implementación de un sistema de recuperación de pérdidas físicas en el primer piso de clarificación, con su respectivo tanque y bombas, $y$

- recuperación de enjuagues dulces en la liquidación de fábrica; en el cual se alcaliniza y se realiza seguimiento con el fin de mantener un pH neutro para evitar la destrucción de la sacarosa.

Adicionalmente, las pérdidas por inversión de sacarosa se controlaron instalando bombas Milton Roy para la dosificación de ácido y sacarato en la refinería.

Las pérdidas generadas en el arrastre de tachos se corrigieron implementando detectores de arrastre en los tachos de refino 1 y 2 ; tachos de blanco 4,5 y 7 y al concentrador de evaporadores. Igualmente, se instalaron separadores de arrastre TJA en los tachos de refino 2 y 3 y un separador de arrastre para el concentrador de los evaporadores de acuerdo con la capacidad de evaporación para $8000 \mathrm{TCD}$.

Otros de los correctivos realizados en los equipos para minimizar la pérdida de sacarosa tuvieron lugar en el equipo de báscula ubicado en la refinería tal como se muestra en la figura 4.

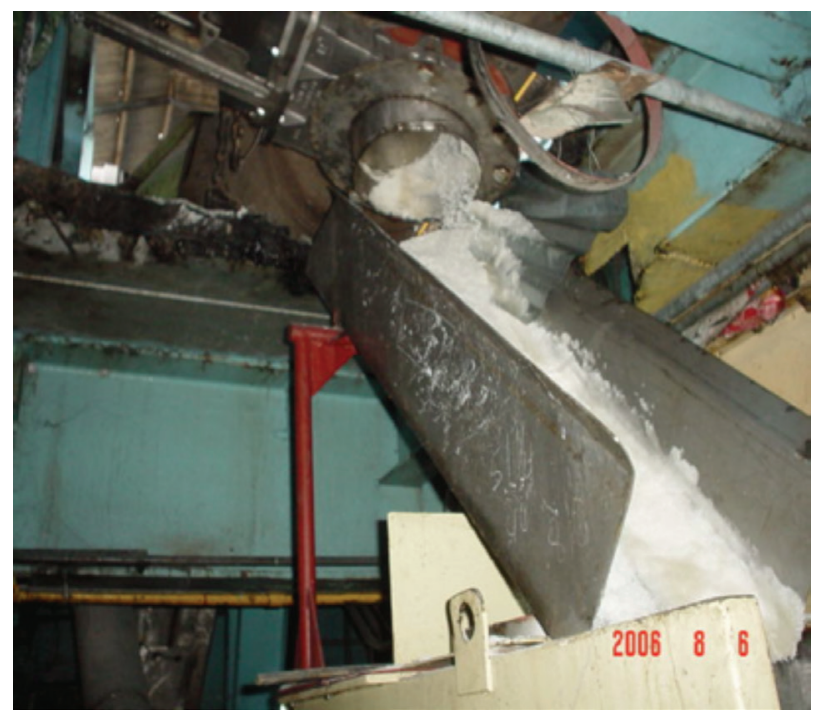

Figura 4. El azúcar puede desbordarse por falta de una tapa Fuente: elaboración propia 


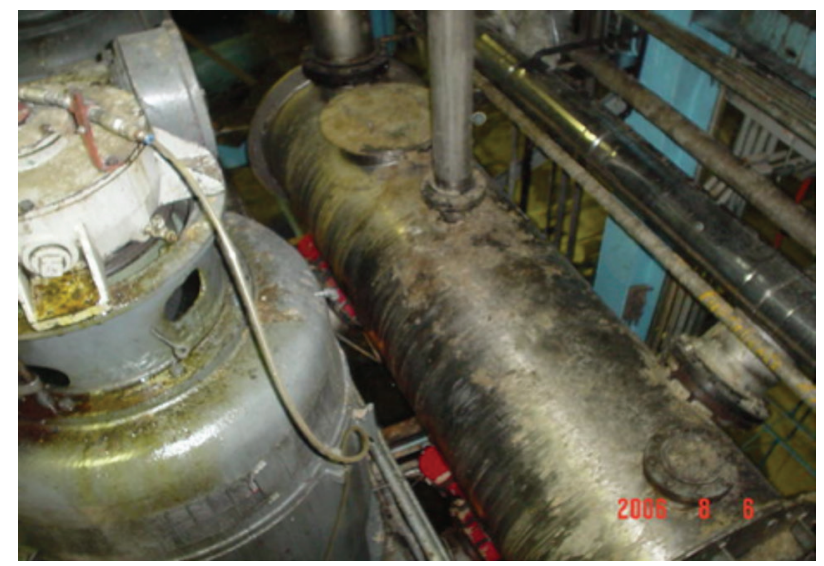

Figura 5. Regueros del material azucarado que caen por fuera del recibidor

Fuente: elaboración propia

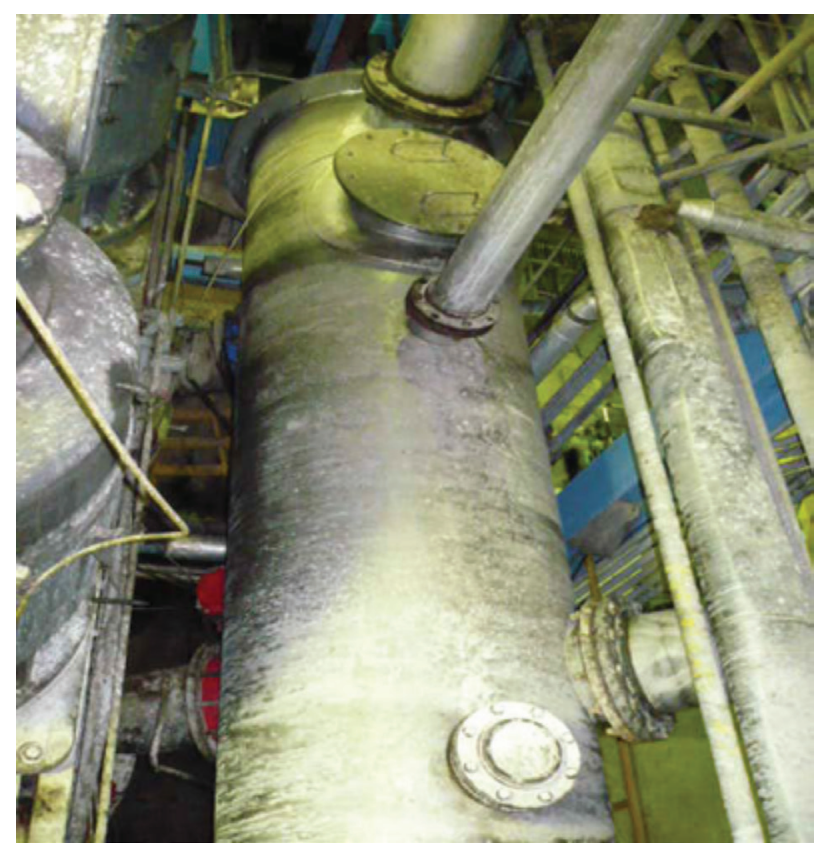

Figura 6. Ausencia de material azucarado sobre el recibidor y motor de la centrífuga

Fuente: elaboración propia

Los equipos zaranda, mezclador, secador y recibidor presentaban inconvenientes relacionados con la filtración del licor, desborde de masa, fuga de masa por eje de transmisión y fugas de azúcar por el acople en la parte móvil, respectivamente, por lo cual también requirieron mejoras en su estructura. Estas mejoras se observan en las figuras 5 y 6 para el equipo mezclador. En la figura 5, se observan los regueros de material azucarado que caían por fuera del recibidor y sobre el motor de la centrifuga de refino, regueros en el mezclador que alimenta a las centrifugas y motores de centrifugas. En la figura 6, se observa la no presencia de material azucarado sobre el recibidor y motor de la centrífuga.

\subsection{Costos de calidad identificados en el proyecto}

El valor económico por fallas internas comprendió el $97,8 \%$ de las pérdidas monetarias que se presentaron por el desperdicio de la sacarosa en la fábrica; estas pérdidas económicas ascendieron a \$ 1728 millones en el periodo 2006-2008 y se presentaban por desechos, trabajo de reelaboración, doble ensayo, tiempos perdidos, bajos rendimientos, altas pérdidas totales y gastos de disposición. Entre tanto, los costos de evaluación representaron el $0,6 \%$ de las pérdidas económicas con un valor de \$10 287623 y los costos de prevención asociados al control diario y rutinario por parte de inspectores y mantenimiento global de la fábrica, representaron un 1,65\% de las pérdidas económicas por año con un valor de \$28655640.

Los porcentajes anteriores muestran el impacto de las fallas internas y la importancia de asignar un presupuesto adecuado para atender la evaluación y la prevención, observándose una disminución en los costos por fallas internas. A mayor inversión en evaluación y prevención, menores son los costos por fallas internas.

\subsection{Impactos logrados}

El primer impacto y el más notable es el económico. Los resultados del cálculo de las pérdidas económicas ocasionadas por las pérdidas de sacarosa en el efluente final de elaboración, demuestran el cumplimiento de los objetivos de este trabajo de investigación, pues se redujeron considerablemente las pérdidas totales e indeterminadas en los dos últimos años como se observa en la tabla 4, que muestra la tendencia de su comportamiento. 
Tabla 4. Impacto económico del proyecto de investigación

\begin{tabular}{|l|c|c|c|c|c|c|c|}
\hline AÑO & $\begin{array}{c}\text { Pérdidas } \\
\text { totales } \\
\text { sacarosa } \\
\text { \%caña }\end{array}$ & $\begin{array}{c}\text { Pérdidas } \\
\text { indeterminadas } \\
\text { sacarosa \%caña }\end{array}$ & $\begin{array}{c}\text { Pérdidas } \\
\text { efluente } \\
\text { sacarosa \%caña }\end{array}$ & Azúcar en qq & $\begin{array}{c}\text { Pérdidas } \\
\text { en pesos } \\
\text { (millones) }\end{array}$ & $\begin{array}{c}\text { Reducción de } \\
\text { pérdidas de } \\
\text { sacarosa en \% }\end{array}$ & $\begin{array}{c}\text { Reducción } \\
\text { de pérdida } \\
\text { económica } \\
\text { en pesos } \\
\text { (millones) }\end{array}$ \\
\hline $\mathbf{2 0 0 6}$ & 2,194 & 0,617 & 0,198 & 37021 & 1777 & & \\
\hline $\mathbf{2 0 0 7}$ & 1,585 & 0,126 & 0,054 & 22680 & 1089 & 71 & 688 \\
\hline $\mathbf{2 0 0 8}$ & 1,444 & 0,049 & 0,003 & 1030 & 49,4 & 93 & 1040 \\
\hline
\end{tabular}

Fuente: elaboración propia

La tabla 4 muestra la notable disminución de las pérdidas de sacarosa; durante 2006 y 2007 la reducción fue del $71 \%$, y entre 2007 y 2008, la reducción fue del $93 \%$ en sacarosa; lo cual significó un ahorro económico de 1728 millones de pesos entre los años 2006 y 2008.

Otro impacto logrado es de carácter social, al obtenerse como resultado del proyecto la creación de seis nuevos puestos de trabajo como Inspector de Pérdidas (tres en la planta Castilla y tres en la planta Riopaila). Finalmente, se alcanzó un impacto técnico al lograrse un mejor manejo y control de la sacarosa en el efluente final y el mejoramiento del sistema de recuperación de aguas dulces.

\section{Conclusiones}

- Las pérdidas se deben básicamente al mal estado de los canales de recuperación y de algunos equipos del proceso.

- Los resultados estadísticos, tanto de las pérdidas totales como de las pérdidas indeterminadas, muestran la importancia del estudio de las pérdidas de sacarosa por efluente final de elaboración.

- El control de las pérdidas físicas de sacarosa en el ingenio es eficiente si se mide por los resultados de pérdidas encontradas en el efluente de la fábrica. Dicho control es útil para el laboratorio, molienda y elaboración, como también para la fábrica en general.

- El método indicado para determinar bajas concentraciones de sacarosa en aguas, es el análisis cuantitativo que emplea fenol -ácido sulfúrico. Sólo por excepción, en materiales o soluciones que visualmente marcan alta sacarosa o alto Brix la medición de la sacarosa presente se debe realizar por el método de polarización.
- En la cuantificación de las pérdidas económicas, en los últimos diez años, se estima que la pérdida promedio ascendía a 1540 millones de pesos por año.

\section{Referencias}

[1] M.H. Guzmán Jaimes, Identificación de las principales pérdidas de sacarosa y estrategias a implementar para aumentar la eficiencia en el proceso de elaboración del azúcar. [En línea] Disponible en: http://prezi.com/ cifiw2qpavcv/identificacion-de-las-principales-perdidas-de-sacarosa-y/

[2] J.E. Laharrondo, "Definición y alcances de la alcoquímica: la calidad de las materias primas y su impacto en el proceso alquímico". Presentado en Congr. AETA. Sept. 1820 2013. [En línea]. Disponible en: http://www.aeta.org. ec/pdf/fabrica/Larrahondo,\%20J.\%20Definicion\%20 y\%20alcances\%20de\%20la\%20alcoquimica.pdf

[3] Centro de Investigación de la Caña de Azúcar en Colombia, CENICAÑA. Disponible en: http://www.cenicana. org/quienes_somos/index.php

[4] H. H. Mosquera, G. Garzón y J. Rubio, “Evaluación, análisis y cuantificación de pérdidas de sacarosa de miel final en los procesos de cristalización, agotamiento y centrifugación del ingenio María Luisa”, Ingenium, vol. 6, n. ${ }^{\circ}$ 12, pp. 69-75, 2012. [En línea]. Disponible en: http://revistas.usc.edu.co/index.php/Ingenium/article/ view/56/48\#.VEZsz_mG9ps

[5] E. R. Zepeda Guardado, "Propuesta de alternativas para la reducción de pérdidas de sacarosa de un ingenio azucarero", Tesis de grado, Esc. Ing. Quim, Universidad de El Salvador, San Salvador, 2012. [En línea]. Disponible en: http://ri.ues.edu.sv/1647/1/TESIS-PROPUESTA_ DE_ALTERNATIVAS_DE_REDUCCI\%C3\%93N_ DE_P\%C3\%89RDIDAS_DE_SACAROSA.pdf

[6] Z. T. Daza, J. A. Martínez, L. M. Calero, J. Laharrondo, M. L. Guzmán y E. F. Castil, "Participación en la acción microbiológica en las pérdidas indeterminadas de sacarosa”, Memorias del VIII Congreso de Cenicañal, 
2009, pp. 655-673. [En línea]. Disponibe en: http:// www.cenicana.org/pdf/otros/eventos/tecnicana/2009/ tabla_contenido_memorias_viii_congreso.pdf

[7] J. H. Payne, "Sugar Cane Factory Analytical Control”, in The Official Methods of the Hawaiian Sugar Technologists, Elsevier: New York, 1968.
[8] P. Rein, P. Turner y K. Mathias, Good Management Practices Manual for The Cane Sugar Industry (Final). 2011. [Online]. Available in: http://www.ifc.org/wps/wcm/con nect/486cf5004953685e8586b519583b6d16/IFC_GMP_ ManualCaneSugarIndustry.pdf?MOD=AJPERES 